Boise State University

ScholarWorks

3-1-2009

Impact of Pedometer Use and Self-Regulation Strategies on Junior High School Physical Education Students' Daily Step Counts

Jane Shimon

Boise State University

Linda M. Petlichkoff

Boise State University 


\title{
Impact of Pedometer Use and Self-Regulation Strategies on Junior High School Physical Education Students' Daily Step Counts
}

\author{
Jane M. Shimon and Linda M. Petlichkoff
}

\begin{abstract}
Background: The aim of this study was to determine the impact of pedometer use and self-regulation strategies on adolescents' daily physical activity. Methods: Junior high school students $(\mathrm{n}=113)$ enrolled in seventh- and eighth-grade physical education classes (52 girls, 61 boys) volunteered to participate in a 5 -week study to assess daily step counts. Ten physical education classes were randomly assigned to 1 of 3 groups: (a) self-regulation, (b) open, and (c) control. Results: A repeatedmeasures, mixed-model analysis of variance revealed a significant $3 \times 4$ (Group by Time) interaction effect, $F_{6,290}=2.64, P<.02$. Followup analyses indicated participants in the selfregulation group took 2071 to 4141 more steps/d than the control. No other significant differences emerged among groups on step counts. Conclusions: It appears that having access to and charting daily step counts (ie, self-regulatory strategies) positively influenced young adolescents to attain a higher number of steps/d.
\end{abstract}

Keywords: pedometry, physical activity, physical education, adolescent

Physical activity has declined, the percentage of body fat has risen, and risk factors associated with cardiovascular disease are evident in children, as well as adults. Over the past 40 years, there has been a $17 \%$ to $19 \%$ increase in overweight children and adolescents. ${ }^{1}$ These percentages support the notion that many children and adolescents' activity levels are insufficient or

The authors are with the Dept of Kinesiology, Boise State University, Boise, ID 83725-1710. below recommended levels (ie, minimum of 30 minutes of moderate activity) for health benefits. ${ }^{2}$ Research also suggests that as children advance into adolescence, their physical activity levels appear to decline. ${ }^{3-6}$

In an effort to assess physical activity levels in children and adolescents during physical education and nonschool hours, researchers have used pedometers to monitor daily steps. ${ }^{7-13}$ Preliminary daily step guidelines indicate that girls should attain 11,000 to 12,000 steps/d and boys should accumulate between 13,000 to 15,000 steps/d. ${ }^{14,15}$ Pedometers also provide feedback to users in an effort to maintain or increase the number of steps taken each day and potentially motivate them to become more active. ${ }^{16}$ In addition to the use of pedometers as a motivational medium to become physically active, self-regulation strategies (eg, self-monitoring) have been shown to enhance motivation when doing a variety of sport and physical activities. ${ }^{17-19}$

Self-regulation is a process whereby individuals assume responsibility for learning by self-monitoring their progress and using strategies to work toward selfimprovement and personal goals. ${ }^{20}$ Self-regulated learners believe they play an instrumental role in their success or whether they achieve their goals (ie, self-directed behavior). Furthermore, self-regulated learning theorists emphasize the importance of self-oriented feedback and stress that feedback is a cyclical process that relies not only on what children and adolescents learn from employing various strategies (ie, how effective those strategies were), but also on how they use feedback to change their behaviors. ${ }^{20,21}$

In the initial phases of self-regulated learning, individuals develop awareness through self-observation (ie, self-monitoring, self-recording), which initiates a goalsetting process. Petlichkoff ${ }^{22}$ suggests such strategies help individuals see what they are currently doing (ie, how physically active they are) so they can set goals for self-improvement (ie, increase step counts). It has been recommended that physical education teachers include 
self-management skills in their lessons to help educate and inform elementary students with regard to current levels of physical activity as measured with pedometers. ${ }^{19}$ Researchers identified specific self-management skills teachers should implement to assess activity levels of students and included such skills as self-assessment, setting personal goals, charting progress, and selfreflection. They emphasized that students became aware of or knowledgeable about current physical activity levels so they could set goals to maintain or increase their physical activity for better health. More importantly, Morgan et al 19(p.36) argued "that learning about physical activity and developing self-management skills are more important than the actual number of steps children accumulate." This information can assist with setting and resetting goals that may lead to more active children.

As indicated earlier, numerous studies have measured physical activity levels of school-age children using pedometers; however, there is limited research on pedometer use with junior high school students. , $^{7,10,23}$ Hence, the purpose of this investigation was to assess the effects of an intervention protocol that used pedometers and self-regulation strategies (ie, self-monitoring and charting) on junior high school students' daily step counts. Specifically, it was hypothesized that students who were able to view and graphically plot daily step counts (ie, self-regulate) would take more steps than those students who simply viewed and reported daily step counts or did not see their daily steps at all.

\section{Methods}

\section{Participants and Setting}

An urban junior high school (grades 7 to 9) located in the northwest region of the United States was selected as the site for this study. The school was composed of approximately $89 \%$ Caucasian students, with $10.5 \%$ of the population being English language learners and $37.8 \%$ receiving free and/or reduces lunches. Participants were predominantly white, non-Hispanic youth. One hundred ninety-four students, ranging in age from 12 to 14 years $($ mean $=13.15, \mathrm{SD}=0.72)$, volunteered to participate in a 5-week study. Students (101 girls, 93 boys) participated as part of their daily seventh- and eighth-grade physical education classes during the months of April and May. Of the 194 students who volunteered to participate, 159 completed the 5-week investigation and represented an $81.9 \%$ retention rate.

\section{Measures}

The Yamax SW-200 (DW200) pedometer was used to monitor daily step counts. Yamax pedometers provide reliable measures of step counts and have been used frequently in pedometer research with adolescents. . $^{7,10,23}$
All pedometers used in this study were new and came to within 1 shake-count of 100 actual shakes. ${ }^{24}$

\section{Procedures}

After permission to conduct the study was obtained from the school principal, a formal proposal was submitted and approved by the university's Institutional Review Board and the school district's research committee. Once parental consent and participants' assent forms were secured, numbered pedometers were distributed during physical education classes. Students were introduced to pedometers and instructed on proper wear and care as specified by the manufacturer. Students were directed to secure the pedometer on their waistband, in line with the right thigh and patella. They were shown how to open and close the device, along with resetting the pedometer back to zero before partaking in walking practice trials. Once students demonstrated proper pedometer use, devices were set to zero, sealed using a large sticker, and returned to students. Pedometers were sealed to prevent students from potentially taking more steps (ie, reactivity) as a result of viewing their pedometers. ${ }^{25}$ Students were told to wear pedometers all day long, removing them only for specific circumstances such as taking a shower, going swimming, athletic contests that prohibited them from wearing external objects on uniforms, and going to bed. They also were given tips on ways to remember to put their pedometer on each morning such as placing the device by their book bag or putting the pedometer in their shoes to be worn at school.

During the course of this investigation, no attempt was made to alter daily physical education class curricula. Seventh- and eighth-grade physical education teachers followed their normal grade-specific lesson plans for each class period throughout the day. This procedure allowed for a degree of consistency in step counts accumulated during physical education classes within each grade level and across pedometer groups.

\section{Baseline}

Daily step counts were monitored over 4 consecutive days to (1) establish an average baseline step count for each participant and (2) determine whether there were any significant differences in daily step counts before implementation of the intervention protocol for group assignments. Research has shown that 4 consecutive days of pedometer data were needed for reliable estimates of habitual physical activity in adolescents. . $^{8,10}$ Participants were unaware of group assignments during baseline data collection.

At the beginning of physical education classes each Monday, numbered pedometers were distributed to students for the week. Then on Tuesday through Friday at the beginning of each class, the physical education 
teacher or student aid removed the seal and recorded daily step counts, set the pedometer back to zero, and resealed it with another sticker. If the seal was broken on any pedometer by anyone other than the physical education teacher or designated aid, no daily step count was recorded for that individual. Pedometers were returned to each participant for another 24-hour period of data collection. On Fridays, step counts were recorded and pedometers were collected and stored until the following Monday.

\section{Intervention}

Once daily step count baseline measures were established for each group, the 4-week intervention phase of the study commenced. Each class period was randomly assigned to 1 of 3 groups so that all students in one class period received the same intervention protocol. Students adhered to one of the following group protocols (see Table 1):

Self-regulation group-This group consisted of 2 seventh- and 2 eighth-grade classes. Students in this group wore nonsealed pedometers and recorded daily step counts at the beginning of physical education class (Tuesday through Friday) on a weekly recording form, as well as plotted their steps on a chart at the start of each class day. Charts were individualized depending on participants' baseline values and started at 5000, 9000 , or 13,000 steps, respectively. Each chart advanced by increments of 500 steps with an 11,000-step allowance along the $y$-axis and days of the week on the $x$-axis. Once data were recorded, students reset their pedometers to zero and began another day of step-count monitoring. Students were told they could open the pedometer and view their step counts at any time during the day; however, they were instructed not to reset the pedometer back to zero. Doing so would erase all the steps they had accumulated for that day. In addition to self-monitoring data, students in the self-regulation group participated in a short (ie, 10-minute) informal discussion session on effective goal-setting strategies during the second week of the intervention. During this discussion, emphasis focused on the importance of setting goals to increase daily step counts. Students described ways to increase daily step counts such as going for a walk with a friend after school, taking their dog for a walk, or walking to school rather than getting a ride. Although students were asked to establish personal daily step-count goals, they were not required to set or write down their goals (an advanced self-regulation strategy).

Open group-This group consisted of 1 seventhand 2 eighth-grade classes. These students were instructed to wear nonsealed pedometers throughout the day, which allowed them to view their step counts any time throughout the day. Participants in the open group recorded their daily steps on a weekly reporting form at the beginning of physical education class; then, they reset their pedometer to zero for another 24-hour recording period. As with the self-regulation group, students in the open group were told not to reset their pedometer at any time during the day.

Control group-This group consisted of 2 seventhand 1 eighth-grade classes. Students wore sealed pedometers over the course of this study and never had the opportunity to view their daily step counts. Physical education teachers or their assistants removed the seal at the beginning of physical education class, recorded steps, set the pedometer back to zero, and applied a new sticker to each pedometer for participants in the control group.

\section{Data Treatment and Analyses}

Data Treatment. Given the potential for student absenteeism from school, misplaced or forgotten pedometers, and/or students electing to drop out, incomplete weekly data sets were anticipated. Of the 159 students who completed the study, it was determined a priori to include students' data only if they missed no more than 1 day per week during the 4-week intervention period (ie, 1 day in a 4-day recording period). For those students who fit the above criteria but had missing data, an individual-centered, data-replacement method based on a student's average step count was used ${ }^{26}$ and resulted in approximately $7 \%$ of the missing data replaced. After data replacement, 113 students (52 girls, 61 boys) met the criteria for usable data. No data were eliminated due to extreme daily step-count scores.

Table 1 Initial and Final Participant Numbers by Group, Grade, and Gender

\begin{tabular}{|c|c|c|c|c|c|c|c|c|}
\hline \multirow[b]{2}{*}{ Characteristics } & \multicolumn{3}{|c|}{ Initial assessment } & \multirow[b]{2}{*}{ Totals } & \multicolumn{3}{|c|}{ Final assessment } & \multirow[b]{2}{*}{ Totals } \\
\hline & $\begin{array}{c}\text { Self- } \\
\text { regulation }\end{array}$ & Open & Control & & $\begin{array}{c}\text { Self- } \\
\text { regulation }\end{array}$ & Open & Control & \\
\hline \multicolumn{9}{|l|}{ 7th grade } \\
\hline boys & 23 & 12 & 19 & $54(28 \%)$ & 13 & 8 & 13 & $33(29 \%)$ \\
\hline girls & 24 & 14 & 22 & $60(31 \%)$ & 15 & 7 & 11 & $34(30 \%)$ \\
\hline \multicolumn{9}{|l|}{ 8th grade } \\
\hline boys & 11 & 17 & 11 & $39(20 \%)$ & 8 & 12 & 7 & $27(24 \%)$ \\
\hline girls & 14 & 17 & 10 & $41(21 \%)$ & 7 & 7 & 5 & $19(17 \%)$ \\
\hline Totals & $72(37 \%)$ & $60(31 \%)$ & $62(32 \%)$ & 194 & $43(38 \%)$ & $34(30 \%)$ & $36(32 \%)$ & 113 \\
\hline
\end{tabular}


Data Analyses. A one-way ANOVA was employed to determine whether there were differences among groups on the 4-day step count measure at baseline. To examine group differences after the 4 -week intervention program, a repeated-measures, mixed-model $3 \times 4$ (Group $\times$ Time) MANOVA was employed. If a significant interaction emerged, specific pairwise comparisons were made within and among groups, and a step-down Bonferroni adjustment was used to adjust the resulting $23 P$ values for multiple comparisons. The $\alpha$ level for all statistical analyses was set at $P=.05$. The initial analyses were fit using SPSS version 12.0 (SPSS, Inc., Chicago, IL), and post hoc comparisons were specified and adjusted with SAS v8.2.

\section{Results}

\section{Baseline}

Intraclass correlation coefficients (ICC) were calculated over 2-, 3-, and 4-day periods to establish a reliable and valid measure of daily step counts for this junior high school sample. Results indicated the 4-day period for monitoring daily step counts (ICC $=.83$ ) met an acceptable criteria for an intraclass coefficient of $.80 .{ }^{26,27}$ The 3-day intraclass correlation coefficient (.79) approached a similar level of acceptability, whereas the 2-day (ICC $=.71)$ fell below this acceptable criteria. Although we felt comfortable using a 3- or 4-day step count measure as a reliable and valid measure of daily steps counts for this sample, we chose to use the 4-day baseline measure to determine whether there were any group differences before the intervention protocol. No significant differences were shown to exist among groups on daily step counts before the intervention protocol, $F_{2,110}=2.06, P$ $=.13$. At baseline, average daily step counts for each of the 3 groups ranged from 11,482 to 13,048 steps per day (Table 2).

\section{Intervention}

A repeated-measure, mixed-model $3 \times 4$ (Group $\times$ Time) MANOVA was employed to examine whether there were differences among groups and across weeks on average daily step counts taken over the 4-week intervention phase. Results revealed significant main effects for groups, $F_{2,110}=6.00, P<.01$, and weeks, $F_{3,290}=7.62, P<.01$, as well as a significant interaction effect for groups by weeks, $F_{6,290}=2.64, P<.02$. Follow-up Bonferroni post hoc analyses revealed that significant differences emerged for the self-regulation and open groups when compared with the control group. On average, the self-regulation group accumulated 3763 to 3883 more daily steps than the control group at weeks 2,3 , and 4 , whereas the open group differed by almost 3510 steps from the control group at week 3 (see Table 2). Although no other group by week differences emerged, daily step counts varied greatly among groups.

Within-group differences were examined to determine whether changes occurred over time for each of the 3 groups. Results indicated the self-regulation group accumulated more steps during weeks 2,3 , and 4 than in week 1; however, a slight nonsignificant decline occurred from week 3 to 4 . For the open group, significant differences in step counts emerged between weeks 2 and 3. No significant differences emerged on daily step counts for the control group over the course of the 4 weeks (see Figure 1).

\section{Post Hoc Analyses}

As anticipated, results indicated that attrition occurred (18.9\%) across groups because of absenteeism, misplaced or forgotten pedometers, or voluntary withdrawal from the study. These levels were comparable to other investigations that have reported attrition rates of $7 \%$ and $28 \%$ for this age group for studies lasting 6 to 14 days. ${ }^{7}, 10$ Although the proportion of participants among treatment groups and grade levels remained similar throughout the study, the number of girls and boys decreased by almost one-half $(49 \%)$ and one-third (34\%) by the end of the study, respectively. Eighthgrade girls had the highest attrition rate (54\%) compared with $45 \%$ for seventh-grade girls. Boys maintained a higher level of participation with a $31 \%$ and $37 \%$ decrease in the eighth- and seventh-grades, respectively.

Table 2 Means (SD) for Step Counts for Group by Week

\begin{tabular}{lccc}
\hline Week & Self-regulation $(\mathbf{n}=\mathbf{4 3})$ & Open $\mathbf{( n = 3 4 )}$ & Control $(\mathbf{n}=\mathbf{3 6})$ \\
\hline Baseline & $13,050(3099)$ & $12,846(4259)$ & $11,483(3562)$ \\
1 & $13,367(3746)$ & $12,407(4227)$ & $11,296(4251)$ \\
2 & $15,508(7400)^{*, a}$ & $12,642(4996)^{\mathrm{b}}$ & $11,745(3495)^{*}$ \\
3 & $15,458(5143)^{*, \mathrm{a}}$ & $15,577(6173)^{* *, \mathrm{~b}}$ & $12,067(3873)^{* * * *}$ \\
4 & $14,974(5427)^{*, \mathrm{a}}$ & $14,568(6356)$ & $11,091(3510)^{*}$ \\
Overall & $14,827(4444)^{*}$ & $13,798(5003)$ & $11,571(3260)^{*}$ \\
\hline
\end{tabular}

Note. Means with same superscripts differ significantly at $P<.05$.

* Differences between self-regulation and control groups.

** Differences between open and control groups.

${ }^{\text {a }}$ Differences from week 1 .

${ }^{\mathrm{b}}$ Differences between week 2 and 3 . 
Moreover, examination of the figures contained in Table 1 suggests that eighth-grade girls in the open group accounted for the largest group loss (59\%), whereas seventh-grade girls in the self-regulation group retained the most participants of all the girls (66\%). Given these attrition rates, data were further analyzed by gender.

\section{Gender}

When participants were compared on step counts taken at baseline by gender, boys took significantly more steps than girls, $F_{1,111}=15.07, P<.01$. On average, boys took 2527 more steps than girls (Table 3); however, when baseline step counts were factored out of the intervention data, a covariate analysis of variance revealed no significant main effect for daily step counts by gender, $F_{1,112}=2.64, P>.10$. Specifically, boys did not differ significantly from girls on daily step counts, even though total daily step counts observed for boys remained anywhere from 2291 to 3314 higher than girls, depending on assigned group.

\section{Discussion}

The purpose of this study was to determine the effects of pedometer use and self-regulation strategies on daily step counts with junior high school students. As hypothesized, individuals in the self-regulation group emerged with higher step counts than those students in the control group; however, self-regulation students did not differ significantly from the open group. The open pedometer group was included to accommodate changes in behavior (ie, increased step counts) that have been shown to occur with adult populations when they wear and view daily step counts. ${ }^{16}$ After further examination of our protocol for the open group, the simple task of students recording daily step count scores on a weekly form may have served as self-referent feedback ${ }^{20,21}$ and resulted in additional step counts. To clearly differentiate between the open and self-regulation groups, it would have been more appropriate to use an alternate reporting mechanism (eg, teachers record daily step counts), rather than self-reporting step counts. In hindsight, this modification would have eliminated the potential self-regulation effects during data collection for the open group and would have focused solely on the behavioral changes that may occur when wearing a pedometer.

Overall, the self-regulation group increased their daily step counts $12 \%$ above their baseline levels, whereas those in the open and control groups increased $7 \%$ and $1 \%$, respectively. In addition, self-regulation participants had a $22 \%$ (3256 steps) increase in steps when compared with their peers who were never allowed to view pedometer steps (ie, control group). This finding equates to approximately 2910 more daily steps for girls and 3933 additional steps for boys. These notable increases in physical activity may have been influenced because students in the self-regulation group became more cognizant of the number of daily steps they took

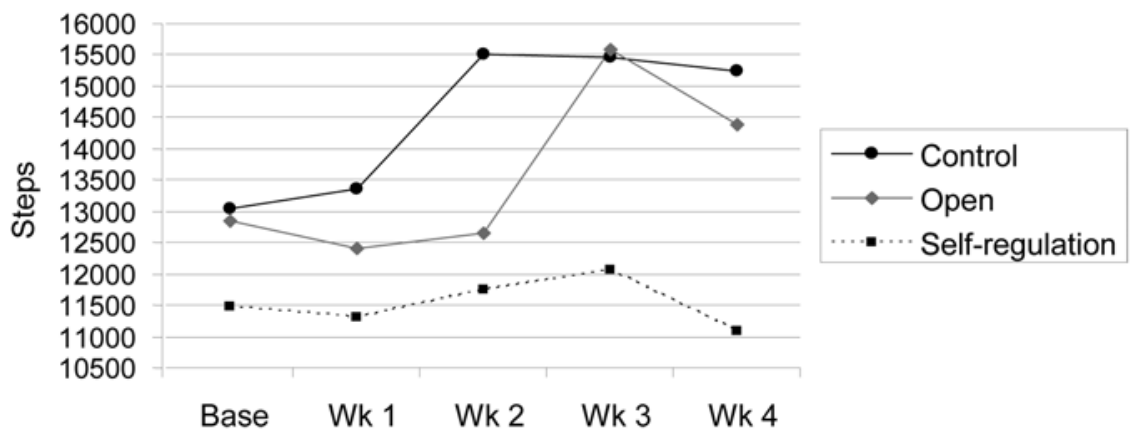

Figure 1 - Interaction effects for groups by weeks on average step counts.

Table 3 Means (SD) for Overall Step Counts at Baseline and Intervention by Group and Gender

\begin{tabular}{lcccc}
\hline Assessment & Self-regulation $(\mathbf{n}=\mathbf{4 3})$ & Open $(\mathbf{n}=\mathbf{3 4})$ & Control $(\mathbf{n}=\mathbf{3 6})$ & Total \\
\hline Baseline & & & & \\
$\quad$ boys & $14,657(2812)$ & $13,982(4238)$ & $12,265(3651)$ & $13,652(3684)^{\mathrm{a}}$ \\
$\quad$ girls & $11,512(2572)$ & $11,222(3869)$ & $10,504(3298)$ & $11,124(3508)$ \\
Intervention & & & & \\
$\quad$ boys & $16,522(3656)$ & $14,568(6356)$ & $11,091(3510)$ & \\
$\quad$ girls & $13,208(4600)$ & $11,947(4842)$ & $10,299(2700)$ & \\
\hline
\end{tabular}


based on the visual cues they received from plotting steps on their charts (ie, self-monitoring strategies). It also is plausible that the short discussion on goal-setting strategies allowed students in the self-regulation group during week 2 to set personal goals to increase daily step counts. This notion fits well with the self-regulation literature, in that, self-regulated learners rely heavily on self-referent feedback to make changes in their behaviors. ${ }^{17,18}$ Students in the open group also took 2227 more steps per day than those individuals in the control group who could not view their pedometer steps. The simple task of opening the pedometer to view step counts throughout the day appeared to provide sufficient information to increase their daily physical activity from baseline values. Overall, those students who were exposed to basic self-regulation strategies or who were able to check their daily pedometer counts increased daily step counts over baseline values.

When observing changes that occurred week by week, the step counts reported at the conclusion of week 1 for the self-regulation and open groups were expected. At the end of week 1, no real step count differences emerged compared with baseline values. Students in these 2 groups were given no other directions during the first week of the intervention other than to record and/or plot daily step counts each day in physical education class. At the beginning of week 2, the self-regulation group was provided with general goal-setting strategies that may explain the large increase in daily step counts for that week. An anomaly emerged at week 3 , when the open group accumulated more steps than members of the self-regulation group; however, this difference was not significant. After thorough review of our design protocol, there were no obvious reasons for this occurrence. There were no changes in physical education lessons because daily curricular activities were similar across grade levels, and we have no evidence to suggest that the physical education teachers discussed goal-setting strategies or step count recommendations with any members of the open group.

Another explanation could be attributed to the attrition rate of the open group. The highest dropout rate of all groups occurred with eighth-grade girls in the open group, which dramatically changed the proportion of boys to girls in this group and may have inflated the daily step counts. It is unknown, however, whether this decline in the number of girls affected the open group's average step counts precisely during week 3 of the study. By week 4, it appeared students in the self-regulation group were able to maintain a higher level of steps compared with the open group. One explanation forwarded for this finding is that the self-monitoring strategy of charting their progress may have aided young adolescents to sustain higher step counts over time compared with those students who only record daily steps.

Several post hoc comparisons were used to examine pedometer step counts based on attrition and gender. The most notable loss occurred for eighth-grade girls, with participation levels dropping 54\%. Anecdotal evidence suggests that girls quit in groups and provided reasons for quitting as they turned in their pedometers such as "Pedometers are stupid," "They don't match my outfit," "They won't stay on my belt," "I can't remember to wear it every day." It may be plausible that those students who consistently wore their pedometers throughout the study were routinely more active or motivated to become more active than those students who missed too many recording periods or quit the study. Further investigations need to account for why students do not participate or quit physical activity programs, especially for girls. Such information would be meaningful and may provide additional insight into adolescent physical activity given the decline in physical activity that tends to occur around age $14 .^{6}$

Another finding reaffirmed the notion that boys are more active than girls. ${ }^{7,8,10,11}$ Pedometer baseline step counts, when compared by gender, indicated that boys took significantly more steps/d $(13,684)$ than girls $(11,124)$. More importantly, boys averaged 2291 to 3314 more steps than girls, irrespective of assigned groups. It is interesting to note, however, that when gender differences were compared using average step counts attained during the intervention phase, no significant gender differences in step counts were found. It may be inferred that self-monitoring and reporting steps had a positive impact on girls' daily step counts, especially for seventh-grade girls.

Girls and boys met minimum step count guidelines for school-age children. ${ }^{12-14}$ This sample of seventhand eighth-grade students who participated in daily physical education were within suggested daily step counts for girls $(11,000$ to 12,000$)$ and boys $(13,000$ to $15,000)$. More important, as a result of the intervention phase, girls in the self-regulation group were above 13,000 steps and boys who participated in the open and self-regulation groups attained at least 15,000 daily steps. Both girls and boys who recorded their daily step counts and/or plotted their step counts on graphs realized an increase in activity compared with those who wore sealed pedometers during the intervention.

In summary, self-regulation strategies (ie, selfmonitoring and self-reporting) had a positive impact on junior high school students to attain a higher number of step counts. In particular, graphing step counts on a chart may have helped girls increase the number of steps attained each day, especially in the seventh grade. Further studies are needed to determine whether employing additional self-regulation strategies (eg, daily and weekly goal setting) enhance daily step counts and physical activity levels with junior high school students.

\section{Acknowledgments}

This study was supported with matching equipment grants from the Idaho Orthopaedic Institute at Saint Alphonsus 
Hospital and the Department of Kinesiology at Boise State University, Boise, ID.

\section{References}

1. National Center for Health Statistics. Health, United States, 2007 with chartbook on trends in health of Americans. http://www.cdc.gov/nchs/hus.htm. Accessed May 20, 2008.

2. US Department of Health and Human Services. Healthy People 2010. April, 2008, ed. http://www.wonder.cdc. gov/data2010/focus.htm. Accessed May 20, 2008.

3. Caspersen CJ, Pereira MA, Curran M. Changes in physical activity patterns in the United States, by sex and crosssectional age. Med Sci Sports Exerc. 2000;32(9):16011609.

4. Pate RR, Trost SG, Dowda M, et al. Tracking of physical activity, physical inactivity, and health-related physical fitness in rural youth. Pediatr Exerc Sci. 1999;11:364376.

5. Trost SG, Pate RR, Sallis JF, et al. Age and gender differences in objectively measured physical activity in youth. Med Sci Sports Exerc. 2002;34:350-355.

6. US Department of Health and Human Services. Physical Activity and Health: A Report of the Surgeon General. Atlanta, GA: Centers for Disease Control and Prevention; 1996. http://www.cdc.gov/nccdphp/sgr/contents. htm. Accessed May 20, 2008.

7. Flohr JA, Todd MK, Tudor-Locke C. Pedometer-assessed physical activity in young adolescents. Res $Q$ Exerc Sport. 2006;77(3):309-315.

8. Le Masurier GC, Beighle A, Corbin CB, et al. Pedometer-determined physical activity levels of youth. J Phys Act Health. 2005;2:159-168.

9. Scruggs PW, Beveridge SK, Eisenman PA, Watson DL, Shultz BB, Ransdell LB. Quantifying physical activity via pedometry in elementary physical education. Med Sci Sports Exerc. 2003;35(6):1065-1071.

10. Le Masurier GC, Corbin CB. Steps counts among middle school students vary with aerobic fitness level. Res $Q$ Exerc Sport. 2006;77(1):14-22.

11. Wilde BE, Corbin CB, Le Masurier GC. Free-living pedometer step counts of high school students. Pediatr Exerc Sci. 2004;16:44-53.

12. Zizzi S, Vitullo E, Rye J, et al. Impact of a three-week pedometer intervention on high school student' daily step counts and perceptions of physical activity. Am J Health Educ. 2006;37(1):35-40.

13. Vincent DS, Pangrazi, RP. An examination of the activity patterns of elementary school children. Ped Exerc Sci. 2002;14(4):432-441.

14. Tudor-Locke C, Pangrazi RP, Corbin CB, et al. BMI-referenced standards for recommended pedometer-determined steps/day in children. Prev Med. 2004;38:857864.
15. Rowlands AV, Eston RG. Comparison of accelerometer and pedometer measures of physical activity in boys and girls, ages 8-10 years. Res $Q$ Exerc Sport. 2005;76(3):251-257.

16. Freedson PS, Miller K. Objective monitoring of physical activity using motion sensors and heart rate. Res $Q$ Exerc Sport. 2000;71:21-29.

17. Crews DJ, Lochbaum MR, Karoly P. Self-regulation: concepts, methods, and strategies in sport and exercise. In: Singer RN, Hausenblas HA, Janelle CM, eds. Handbook of Sport Psychology. New York: John Wiley Son; 2001:566-603.

18. Gould D, Chung Y. Self-regulation skills in young, middle, and older adulthood. In: Weiss MR, ed. Developmental Sport and Exercise Psychology: A Lifespan Perspective. Morgantown, WV: Fitness Information Technology Inc; 2004:383-402.

19. Morgan CF, Pangrazi RP, Beighle A. Using pedometers to promote physical activity in physical education. J Phys Educ Rec Dance. 2003;(7):33-38.

20. Zimmerman BJ. Attaining self-regulation: a social cognitive perspective. In: Boekaerts M, Pintrich PR, Zeidner M, eds. Handbook of Self Regulation. San Diego, CA: Academic Press; 2000:13-39.

21. Zimmerman BJ. Models of self-regulated learning and academic achievement. In: Zimmerman BJ, Schunk DH, eds. Self-Regulated Learning and Academic Achievement: Theory, Research, and Practice. New York, NY: Springer-Verlag; 1989:1-25.

22. Petlichkoff LM. Self-regulation skills for children and adolescents. In: Weiss MR, ed. Developmental Sport and Exercise Psychology: A Lifespan Perspective. Morgantown, WV: Fitness Information Technology Inc; 2004:269-288.

23. Rowe DA, Mahar MT, Raedeke TD, Lore J. Measuring physical activity in children with pedometers: reliability, reactivity, and replacement of missing data. Pediatr Exerc Sci. 2004;16:343-354.

24. Vincent SD, Sidman CL. Determining measurement error in digital pedometers. Meas Phys Educ Exerc Sci. 2003;7(1):19-24.

25. Vincent DS, Pangrazi RP. Does reactivity exist when measuring activity levels with pedometers? Pediatr Exerc Sci. 2002;14:56-63.

26. Baranowski M, de Moor C. How many days was that? intra-individual variability and physical activity assessment. Res Q Exerc Sport. 2000;71(2):74-78.

27. Nunnally J, Bernstein IH. Psychometric Theory. 3rd ed. Boston, MA: McGraw-Hill; 1994. 
Copyright of Journal of Physical Activity \& Health is the property of Human Kinetics Publishers, Inc. and its content may not be copied or emailed to multiple sites or posted to a listserv without the copyright holder's express written permission. However, users may print, download, or email articles for individual use. 
Copyright of Journal of Physical Activity \& Health is the property of Human Kinetics Publishers, Inc. and its content may not be copied or emailed to multiple sites or posted to a listserv without the copyright holder's express written permission. However, users may print, download, or email articles for individual use. 